\title{
Estudio sobre la capacidad técnica y las competencias transversales desarrolladas en las prácticas externas universitarias
}

\author{
Study on technical capacity and transversal competencies \\ developed in university internships
}

\author{
Mercedes Mareque Álvarez-Santullano ${ }^{a}$, Elena de Prada Creo ${ }^{b}$, \\ Margarita Pino Juste \\ a Facultad de Ciencias Empresariales y Turismo, Universidad de Vigo \\ Correo electrónico: chedesmareque@uvigo.es

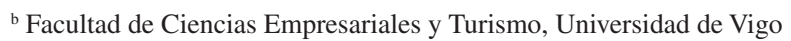 \\ Correo electrónico: edeprada@uvigo.es \\ ${ }^{\mathrm{c}}$ Facultad de Ciencias de la Educación y del Deporte, Universidad de Vigo \\ Correo electrónico: mpino@uvigo.es
}

\begin{abstract}
El objetivo de este estudio es analizar las percepciones de los empleadores y estudiantes universitarios sobre la capacidad técnica y el nivel de adquisición de las competencias transversales desarrolladas a través de las prácticas externas. Además, también se examina el contenido de las memorias de verificación de los grados objeto de estudio con la finalidad de contrastar que incluyen todas las competencias evaluadas en las prácticas externas. Para lo cual se analizan las prácticas externas realizadas por el alumnado de los grados de Ciencias Empresariales y Turismo durante cuatro cursos consecutivos y, se revisan las memorias de verificación de estas titulaciones. Los resultados indican una alta valoración en todas las competencias, destacando el trabajo en equipo. Además, se constata cierta arbitrariedad en el uso de los términos que contienen las memorias de verificación para definir las competencias y en algunos casos incongruencias en su identificación respecto al marco legal vigente.
\end{abstract}

Palabras clave: evaluación de competencias, practicum, educación superior, graduados universitarios, empleadores

\section{ABSTRACT}

The aim of this study is to analyze the employers' and university students' perceptions about the students' technical capacity and level of acquisition of transversal skills established through external internships. In addition, it also examines the content of the degree course specifications to check if they include all the skills evaluated in external internships. For this purpose, the external internships undertaken by the students of the Business and Tourism degrees for a period of 4 consecutive academic years were analyzedand both degree course specifications were reviewed. The results show a very positive assessment for students' transversal skills, especially for teamwork. Additionally, in the analyzed degree course specifications we observe a great deal of arbitrariness in the use of the terms applied to define the different competencies as well as incongruences in their identification based on the existing legal framework.

Key words: competence-based assessment, work placement, higher education, college graduates, employers

Este trabajo fue financiado a través de los Proyectos FONDECYT 3180291, CONICYT + PIA/ Concurso apoyo a centros científicos y tecnológicos de excelencia con financiamiento basal AFB170001 y FONDEF \#ID16I10119. 
Estudios Pedagógicos XLIV, N 3: 137-155, 2018

ESTUDIO SOBRE LA CAPACIDAD TÉCNICA Y LAS COMPETENCIAS TRANSVERSALES DESARROLLADAS EN

LAS PRÁCTICAS EXTERNAS UNIVERSITARIAS

\section{INTRODUCCIÓN: JUSTIFICACIÓN Y MARCO TEÓRICO}

El concepto de empleabilidad, entendido como la aptitud que debe poseer un ciudadano para encontrar un trabajo en relación a las cualificaciones que posee o la relación entre la formación y la vida profesional de cada persona, ha sido uno de los aspectos centrales en el análisis de la calidad de las universidades desde la constitución del Espacio Europeo de Educación Superior (EEES) (Alonso, Fernández \& Nyssen, 2009; Argos \& Ezquerra, 2014; Lantarón, 2014). Cada vez más, las empresas valoran y evalúan a sus empleados a través de competencias, por lo que su importancia, definición y categorización constituye una importante línea de investigación y de trabajo (Mulder, Gulikers, Biemans \& Wesselink, 2009; OCDE, 2002).

A partir de la "Declaración de Bolonia" en 1999, la apuesta por la calidad educativa plantea la mejora del proceso formativo del alumnado y, a su vez, determina de forma muy importante el dominio y desarrollo de sus capacidades clave y, por lo tanto, su formación integral y su inserción profesional (Acebrón, 2008; Alonso et al., 2009; Álvarez-Rojo \& Lázaro, 2002). La incorporación de las competencias a los estudios universitarios resulta un elemento básico para la formación, en una sociedad cambiante que reformula sus demandas constantemente y que a su vez aspira a profesionalizar la formación universitaria acercando la universidad a la sociedad y al mundo laboral (Gallifa \& Garriga, 2010). En este sentido, Agudo, Hernández-Linares, Rico \& Sánchez (2013) señalan que las competencias facilitan el desarrollo de una verdadera educación integral al englobar todas las dimensiones del ser humano (saber, saber hacer y, saber ser y estar) y suponen un referente para la superación de una enseñanza meramente academicista con escasa vinculación con el ámbito laboral.

Dado que la calidad de la educación es fundamental para el éxito económico de un país, es importante determinar si las estrategias y los métodos utilizados en el proceso de enseñanza-aprendizaje son eficaces para el dominio de las competencias diseñadas en cada titulación. En este sentido, el EEES señala la importancia de vincular la formación universitaria con el mundo profesional y define un nuevo marco de competencias profesionales ligado a las nuevas prioridades formativas que deben adquirir los estudiantes para satisfacer las necesidades del mercado laboral (Gil, 2007; Kirkwood-Tucker, 2004). Según Rue (2007), la planificación didáctica que propone el EEES se debe centrar en las competencias que debe adquirir el estudiante, rompiendo el concepto tradicional lineal del profesorado: contenidos, métodos de enseñanza y sistemas de evaluación (Mateo y Vlachopoulos, 2013). De ahí que, el término "competencias" en el ámbito universitario europeo esté ligado al proceso de armonización transparente de los títulos y vincule de manera directa la formación universitaria con el mundo profesional y con la libre circulación de estudiantes.

En España, la normativa actual no ha sido ajena a estas propuestas europeas ya que, al formar parte de los países que se sumaron al plan europeo de convergencia de la educación superior, ha adoptado su normativa (Sánchez-Elvira, López-González \& Fernández-Sánchez, 2010). Tanto la publicación del Documento Marco por el Ministerio de Educación, Cultura y Deporte (MECD, 2003) como la formalización de las nuevas enseñanzas del Real Decreto 1393/2007 (BOE, 2007), que establece la ordenación de las enseñanzas universitarias oficiales, mencionan en lo referente a las competencias y a las nuevas metodologías, que los objetivos formativos deben incluir competencias más 
específicas que ofrezcan orientación profesional y conduzcan a la integración posterior en el mercado de laboral (MECD, 2003).

Así, en los títulos oficiales españoles se distinguen cuatro tipos de competencias: (i) las competencias básicas, comunes a todos los títulos del mismo nivel; (ii) las competencias transversales (o genéricas), comunes a todos los títulos de una misma universidad; (iii) las competencias generales, comunes a todos los títulos de una universidad, pero adaptadas al contexto específico de cada uno de los títulos; (iv) las competencias específicas, propias del título y orientadas a la consecución del perfil específico del egresado. Sin embargo, existe bastante ambivalencia en el manejo de estos conceptos. Clemente-Ricolfe y Escribá-Pérez tratan de precisar estos términos señalando que las competencias profesionales pueden distinguirse en específicas y genéricas indicando que las competencias transversales son competencias genéricas, comunes a la mayoría de las profesiones y que se relacionan con la puesta en práctica integrada de aptitudes, rasgos de personalidad, conocimientos y valores adquiridos (Clemente-Ricolfe \& Escribá-Pérez, 2013).

Por otra parte, desde el ámbito laboral se le da bastante importancia a que los futuros candidatos posean competencias de tipo técnico (entendidas como capacidades técnicas), las cuales se refieren al saber, es decir, a habilidades específicas relacionadas con el correcto desempeño de un ámbito profesional concreto. En general, la mayoría de las competencias son de carácter general y básico y se relacionan directamente con saber aplicar conocimientos en el ámbito profesional y demostrar por medio de la elaboración, de la defensa de argumentos y de la resolución de problemas el dominio de los contenidos del área. Lo deseable sería que un alumno llegase a su etapa profesional provisto de las competencias básicas que posteriormente impulsarán el desarrollo y la adquisición de las competencias específicas (Tejada, 2012).

No obstante, algunos autores han detectado la existencia de una brecha importante entre el ámbito universitario y el laboral, no solo en cuanto a la forma de entender el concepto de competencia sino también en relación a la existencia de una diferencia constatable entre cuales deben ser las competencias adquiridas durante la formación universitaria y las requeridas por el mercado laboral. Esto último ha sido puesto de manifiesto en diversos estudios tanto a nivel internacional (Jackson, 2012; Kellermann, 2007; Velde, 2009) como en el ámbito español (Alonso et al., 2009; Cabrerá, López \& Portillo, 2016; Freire, Teijeiro $\&$ Pais, 2011). Sería importante poder mejorar la relación entre la universidad y la empresa, tratando de adaptar la formación universitaria a las necesidades del mundo laboral. En este sentido, Parris y Saville afirman que habría que trabajar de cara a mejorar tanto la formación práctica del alumnado universitario como su desarrollo competencial, con la finalidad de su mejor adaptación al mundo laboral (Parris \& Saville, 2011). Para ello, se podría recurrir a tres tipos de medidas: realizar más prácticas en empresas, intentar estrechar lazos con el mundo laboral e incrementar en los planes de estudios aspectos vinculados a la práctica profesional. También, en el estudio de Cabrera et al., los empleadores plantean la necesidad de incrementar el tiempo del practicum, revalorizando la importancia de la formación a través de la realización de prácticas externas y de que se tengan en cuenta, a la hora de plantear los planes de estudios, las necesidades reales del desempeño profesional (Cabrera et al., 2016).

Para las titulaciones objeto de estudio en este trabajo, los grados de Administración de Empresas (ADE) y Turismo, existen estudios tanto en el grado de Turismo (Huete, 2007; Munar, 2007) como en ADE (Acedo, Azcona, Riaño \& Ruiz, 2017; Montoro, Mora \& Ortiz 
de Urbina, 2012) que permiten conocer cuáles podrían ser las competencias adecuadas para una formación integral en la enseñanza superior, dada la importancia que la mayoría de los profesionales de estos sectores conceden al período de prácticas en empresas (Freire et al., 2013). Además, cabe destacar que también existen algunos estudios previos que analizan la importancia de las competencias genéricas y específicas de las titulaciones descritas: para el caso de Turismo (López-Bonilla \& López-Bonilla, 2014; Munar \& Montaño, 2009) y para ADE (Arquero, Byrne, Flood \& González, 2009; Azevedo, Apfelthaler \& Hurst, 2012; Jackson \& Chapman, 2012a, 2012b; Lans, Blok \& Wesselink, 2014).

En definitiva, el sistema educativo universitario tiene un importante papel relacionado con la enseñanza de competencias a los alumnos, con la finalidad de que estos futuros profesionales obtengan a lo largo de sus estudios las competencias, capacidades técnicas y habilidades prácticas necesarias que les capacitarán para ejercer de forma óptima una labor profesional. En este sentido, la posibilidad de realización de prácticas externas a lo largo de los estudios universitarios refuerza la formación de los estudiantes, y les permite, por un lado, aplicar las capacidades y conocimientos adquiridos en sus estudios, desarrollando así las competencias profesionales en el ámbito laboral, y por otro, reforzar la adquisición de conocimientos, habilidades profesionales y actitudes, demostrando sus capacidades y sus competencias. Desde el ámbito empresarial, permite a los empleadores comprobar si las competencias adquiridas por el alumnado, como resultado de su formación, se adaptan a las requeridas en los puestos de trabajo (Mareque \& De Prada, 2018).

A este respecto, el objetivo de este estudio es doble, en primer lugar, analizar las percepciones de los empleadores y de los estudiantes universitarios sobre la capacidad técnica y el nivel de adquisición de las competencias transversales en su primera toma de contacto con el mundo laboral, establecida a través de las prácticas externas, con el fin de observar cuales de estas competencias muestran tener un efecto más positivo en el desarrollo de la actividad laboral. En segundo lugar, examinar el contenido de las memorias de verificación de los grados con el fin de comprobar que incluyen todas las competencias evaluadas en las prácticas externas.

La contribución de este estudio se basa en proponer medidas de retroalimentación que permitan dilucidar si la formación competencial adquirida en el ámbito académico y contrastada en el periodo de prácticas en las empresas es percibida de forma positiva tanto por empleadores como por el alumnado o, por el contrario, manifiesta carencias y limitaciones que deben ser subsanadas. En este sentido, los resultados de este estudio desprenden implicaciones que pueden ser interesantes para diversos colectivos. Para los docentes, con la finalidad de que puedan trabajar en sus materias de forma más profunda aquellas competencias más valoradas desde el ámbito empresarial, incorporando actividades prácticas que desarrollen y fomenten tales competencias. Para las instituciones académicas, con el objetivo de que puedan tomar conciencia de las diferencias existentes entre lo que el ámbito empresarial demanda y lo que el ámbito educativo ofrece, tratando de establecer medidas que puedan reducir tal distancia, y acercar el empresariado al entorno educativo. Para el alumnado, con el fin de que conozca cuáles son las competencias más valoradas en el actual mercado laboral pudiendo mejorar aquellas donde tenga algún tipo de carencia. 


\section{METODOLOGÍA}

\subsection{PARTICIPANTES}

La población objeto de estudio está formada por la totalidad de empleadores y alumnado participante en las prácticas académicas externas realizadas durante cuatro cursos académicos en la Facultad de Ciencias Empresariales y Turismo de la Universidad de Vigo. Se analizaron un total de 533 prácticas, donde 319 fueron realizadas por alumnado del grado en ADE y 214 por el alumnado del grado en Turismo. El número de prácticas estudiadas en cada curso es de 141 en 2013-14, 133 en 2014-15, 130 en el 2015-16 y 129 en el 2016-17. En este estudio se analizan, por tanto, un total de 1066 informes de prácticas externas, la mitad cubiertos por los empleadores y la otra mitad por los estudiantes. El 70\% del total de las prácticas fueron realizadas por mujeres.

\subsection{INSTRUMENTO}

La gestión de las prácticas externas en empresas realizadas por el alumnado de la Facultad de Ciencias Empresariales y Turismo se desarrolla bajo un proceso definido por el Área Empleo y Emprendimiento de la Universidad de Vigo, el cual estipula que a la finalización de las prácticas tanto el alumnado como el empleador deben cubrir un informe valorando un conjunto de competencias desarrolladas en las mismas, concretamente: capacidad técnica, administración de los trabajos, habilidades de comunicación oral y escrita, creatividad, iniciativa, motivación y trabajo en equipo. Estos informes son comunes para todas las titulaciones de la universidad.

Para la medición del desarrollo de estas competencias se ha utilizado una escala tipo Likert de 1 a 5 , donde 1 indica "mal" y 5 "excelente".

Además, el instrumento recoge otros datos de carácter informativo que permiten conocer el perfil de la población estudiada como el género del alumnado, el grado que cursa, el curso académico y el número de horas de duración de las prácticas.

Para corroborar la fiabilidad de la escala de medida se calculó el coeficiente del Alfa de Cronbach, cuyos resultados mostraron un coeficiente alfa de 0,898 para la muestra de alumnos y de 0,910 para la muestra de empleadores, lo que implica una alta fiabilidad en ambos casos (Gliem \& Gliem, 2003; Sijtsma, 2009).

\subsection{PROCEDIMIENTO}

Para el análisis de las percepciones de los empleadores y estudiantes sobre la capacidad técnica y el nivel de adquisición de las competencias transversales desarrolladas a través de las prácticas externas, dado que la distribución de las variables de cada una de las competencias analizadas no seguía una distribución normal $(\mathrm{p}=0.0001)$, se opta por el empleo de los estadísticos no paramétricos, utilizando la prueba U de Mann Whitney para establecer las relaciones entre las variables dicotómicas y la prueba de Kruskal Wallis para las variables politómicas. Para las variables escala se utilizará la correlación de Spearman Brown. En todos los contrastes efectuados se asumió un nivel de significación de 0,05. El análisis de datos se llevó a cabo usando el paquete estadístico SPSS (versión 22). 
Estudios Pedagógicos XLIV, N 3: 137-155, 2018

ESTUDIO SOBRE LA CAPACIDAD TÉCNICA Y LAS COMPETENCIAS TRANSVERSALES DESARROLLADAS EN LAS PRÁCTICAS EXTERNAS UNIVERSITARIAS

Con el fin de interpretar los datos de corte cualitativo y poder comprobar el tratamiento de las competencias transversales en las memorias de verificación de los grados que están siendo evaluadas en las prácticas externas, se realizó un análisis de contenido de la información descrita en las citadas memorias de los dos grados analizados, ADE y Turismo.

\section{RESULTADOS}

A continuación, se presentan los resultados más relevantes para los dos objetivos de este estudio.

\subsection{RESULTADOS DEL ANÁLISIS DE LOS INFORMES DE EVALUACIÓN DE PRÁCTICAS EXTERNAS}

\subsubsection{Estadísticos descriptivos}

En la Tabla 1 se aprecian diferencias significativas entre las medias del alumnado y empleadores excepto en habilidades de comunicación oral y escrita $(\mathrm{p}=, 122)$. En todas las demás competencias (capacidad técnica, administración de trabajos, creatividad, iniciativa, motivación y trabajo en equipo) el empleador tiene una percepción más elevada del dominio de las competencias que desarrolla el alumnado durante las prácticas. Asimismo, se puede constatar que todas las medias son relativamente altas ya que se aproximan a la máxima puntuación destacando la capacidad de trabajo en equipo.

Tabla 1. Estadísticos descriptivos sobre las competencias desarrolladas en las prácticas según empleadores y alumnado y, prueba U Mann Whitney para diferencias entre ambos grupos

\begin{tabular}{|c|c|c|c|c|c|c|c|c|}
\hline & & Mín. & Max. & Media & $\begin{array}{l}\text { Desviación } \\
\text { Típica }\end{array}$ & Asimetría & Curtosis & $\begin{array}{c}\text { U Mann } \\
\text { Whitney } \\
\text { Sig. }\end{array}$ \\
\hline \multirow{2}{*}{$\begin{array}{l}\text { Capacidad } \\
\text { técnica }\end{array}$} & $\mathrm{E}$ & 1 & 5 & 4,55 & ,619 & $-1,223$ & 1,619 & \multirow{2}{*}{,001 } \\
\hline & $\mathrm{AL}$ & 1 & 5 & 4,38 & ,751 & $-1,321$ & 2,393 & \\
\hline \multirow{2}{*}{$\begin{array}{l}\text { Administración } \\
\text { de trabajos }\end{array}$} & $\mathrm{E}$ & 2 & 5 & 4,63 & ,565 & $-1,380$ & 1,607 & \multirow{2}{*}{,037 } \\
\hline & $\mathrm{AL}$ & 1 & 5 & 4,52 & ,709 & $-1,416$ & 1,711 & \\
\hline \multirow{2}{*}{$\begin{array}{l}\text { Habilidades de } \\
\text { comunicación } \\
\text { oral y escrita }\end{array}$} & $E$ & 2 & 5 & 4,58 & ,634 & $-1,307$ & ,944 & \multirow{2}{*}{, 122} \\
\hline & $\mathrm{AL}$ & 1 & 5 & 4,50 &, 701 & $-1,293$ & 1,303 & \\
\hline \multirow{2}{*}{ Creatividad } & $\mathrm{E}$ & 1 & 5 & 4,43 & ,689 & $-1,049$ & ,969 & \multirow{2}{*}{,0001 } \\
\hline & $\mathrm{AL}$ & 1 & 5 & 4,00 & ,973 &,- 818 &, 147 & \\
\hline \multirow{2}{*}{ Iniciativa } & $\mathrm{E}$ & 1 & 5 & 4,55 & 677 & $-1,567$ & 2,851 & \multirow{2}{*}{012} \\
\hline & $\mathrm{AL}$ & 1 & 5 & 4,38 & ,884 & $-1,565$ & 2,279 & \\
\hline \multirow{2}{*}{ Motivación } & $\mathrm{E}$ & 2 & 5 & 4,73 &, 517 & $-2,053$ & 4,772 & \multirow{2}{*}{,0001 } \\
\hline & $\mathrm{AL}$ & 1 & 5 & 4,53 &, 810 & $-2,020$ & 4,175 & \\
\hline \multirow{2}{*}{$\begin{array}{l}\text { Trabajo en } \\
\text { equipo }\end{array}$} & $\mathrm{E}$ & 2 & 5 & 4,75 &, 524 & $-2,335$ & 6,225 & \multirow{2}{*}{,045 } \\
\hline & $\mathrm{AL}$ & 1 & 5 & 4,62 & ,752 & $-2,284$ & 5,258 & \\
\hline
\end{tabular}


En el Gráfico 1, se observan las distancias entre las opiniones de empleadores y alumnado en los cuatro años analizados para las siete competencias evaluadas. Como se puede ver el trabajo en equipo es una competencia valorada por los empleadores siempre de la misma forma mientras que en opinión del alumnado ha ido adquiriendo mayor dominio en la misma con el paso de los años. También destaca el alto grado de motivación que observan los empleadores que, aunque es más bajo en opinión del alumnado mantiene valores altos en ambos casos.

La única variable que se mantiene constante y es similar en los cuatro años y además no hay diferencias entre la opinión del alumnado y empleadores son las habilidades de comunicación.

La mayor diferencia en las opiniones se produce con respecto a la creatividad, aunque la opinión del alumnado sobre su dominio ha ido mejorando en los últimos cursos académicos. Es la capacidad en la que se considera que los alumnos tienen menor dominio.

En las demás competencias hay mayor grado de acuerdo y su puntuación es relativamente alta y se mantiene en todos los cursos aumentando, en opinión del alumnado, la iniciativa.

No se constatan diferencias entre las medias de ninguna de las capacidades durante los cuatro años lo que significa que a medida que aumenta la percepción del alumnado sobre sus capacidades también lo hace la del empleador.

Gráfico 1. Evolución de las competencias evaluadas por empleadores y alumnado entre el curso 2013-14 al 2016-17

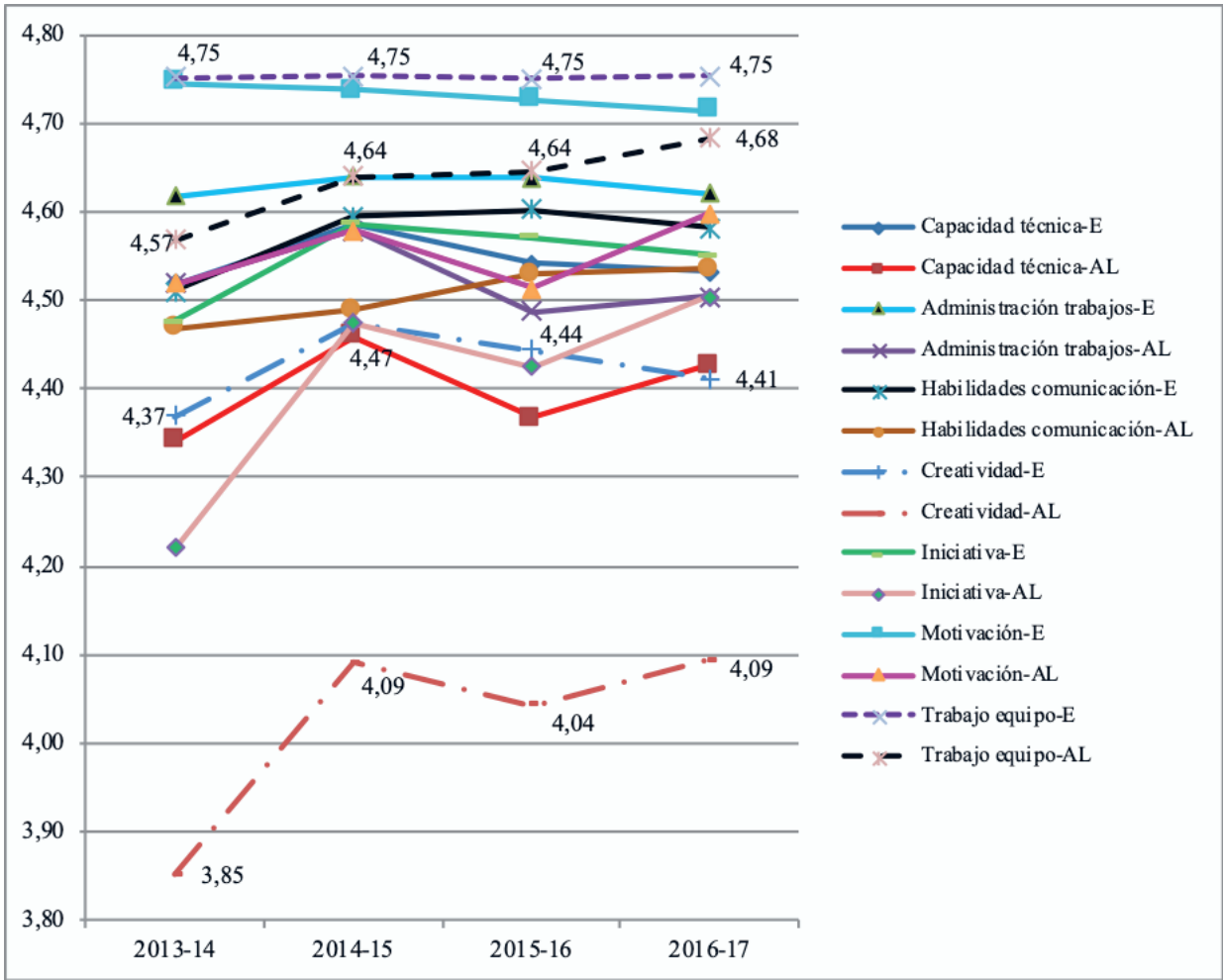

Notas: E: empleador, AL: alumno 


\subsubsection{Correlaciones}

En la Tabla 2 se puede comprobar que existen correlaciones positivas y significativas entre todas las competencias y también entre la opinión del alumnado y de los empleadores, aunque salvo excepciones, las correlaciones son bajas, lo que puede sugerir que estas relaciones no se establezcan de forma lineal. Destaca que las correlaciones más altas se establecen entre las competencias de trabajo en equipo, administración de trabajos, creatividad, iniciativa y motivación tanto en opinión de los empleadores como del alumnado. Sin embargo, la opinión del alumnado no es coincidente con la opinión de los empleadores y aquí disminuyen las correlaciones.

Con respecto a la relación entre el número de horas de las prácticas y las diferentes competencias se encuentra, en el caso de los empleadores, una correlación significativa negativa, aunque baja, entre esta variable y la competencia creatividad. Por ello se podría inferir que los empleadores creen que a más horas de prácticas menor índice de creatividad.

En el caso del alumnado, se observa una correlación positiva entre el número de horas y las habilidades comunicativas, por lo que el alumnado considera que a mayor número de horas de prácticas mayores capacidades en habilidades comunicativas.

\subsubsection{Análisis inferencial}

Para evitar un número excesivo de tablas, hemos incluido solo aquellas en las que se encontraron diferencias significativas.

La primera variable considerada en este análisis es el género (Tabla 3). Solamente se observan diferencias significativas en el caso de los empleadores, en la capacidad de administrar trabajos y en habilidades de comunicación oral y escrita, siendo en ambas variables mayor en las mujeres.

En el caso del alumnado, solamente se hallaron diferencias en la competencia de iniciativa personal, siendo mayor también en las mujeres. Si bien, cabe destacar que, en todas las competencias analizadas, tanto desde la perspectiva del alumnado como del empleador, el rango promedio es siempre superior en las mujeres.

No se encontraron diferencias ni en la opinión del alumnado ni de los empleadores en competencia técnica, creatividad, motivación o trabajo en equipo.

Tabla 3. Resultados de la prueba U Mann-Whitney sobre las competencias desarrolladas según empleadores y alumnado en función del género

\begin{tabular}{|c|c|c|c|c|c|}
\hline & & $\mathbf{N}$ & Rango promedio & Suma rangos & Sig. \\
\hline \multirow[t]{2}{*}{ Administración de trabajos-E } & Hombre & 161 & 250,11 & 40268,50 & \multirow{2}{*}{042} \\
\hline & Mujer & 372 & 274,31 & 102042,50 & \\
\hline \multirow{2}{*}{$\begin{array}{l}\text { Habilidades de comunicación } \\
\text { oral y escrita-E }\end{array}$} & Hombre & 161 & 248,59 & 40023,00 & \multirow{2}{*}{, 030} \\
\hline & Mujer & 372 & 274,97 & 102288,00 & \\
\hline \multirow[t]{2}{*}{ Iniciativa-AL } & Hombre & 161 & 243,14 & 39146,00 & \multirow{2}{*}{,008 } \\
\hline & Mujer & 372 & 277,33 & 103165,00 & \\
\hline
\end{tabular}


Estudios Pedagógicos XLIV, $\mathrm{N}^{\circ}$ 3: 137-155, 2018 ESTUDIO SOBRE LA CAPACIDAD TÉCNICA Y LAS COMPETENCIAS TRANSVERSALES DESARROLLADAS EN LAS PRÁCTICAS EXTERNAS UNIVERSITARIAS

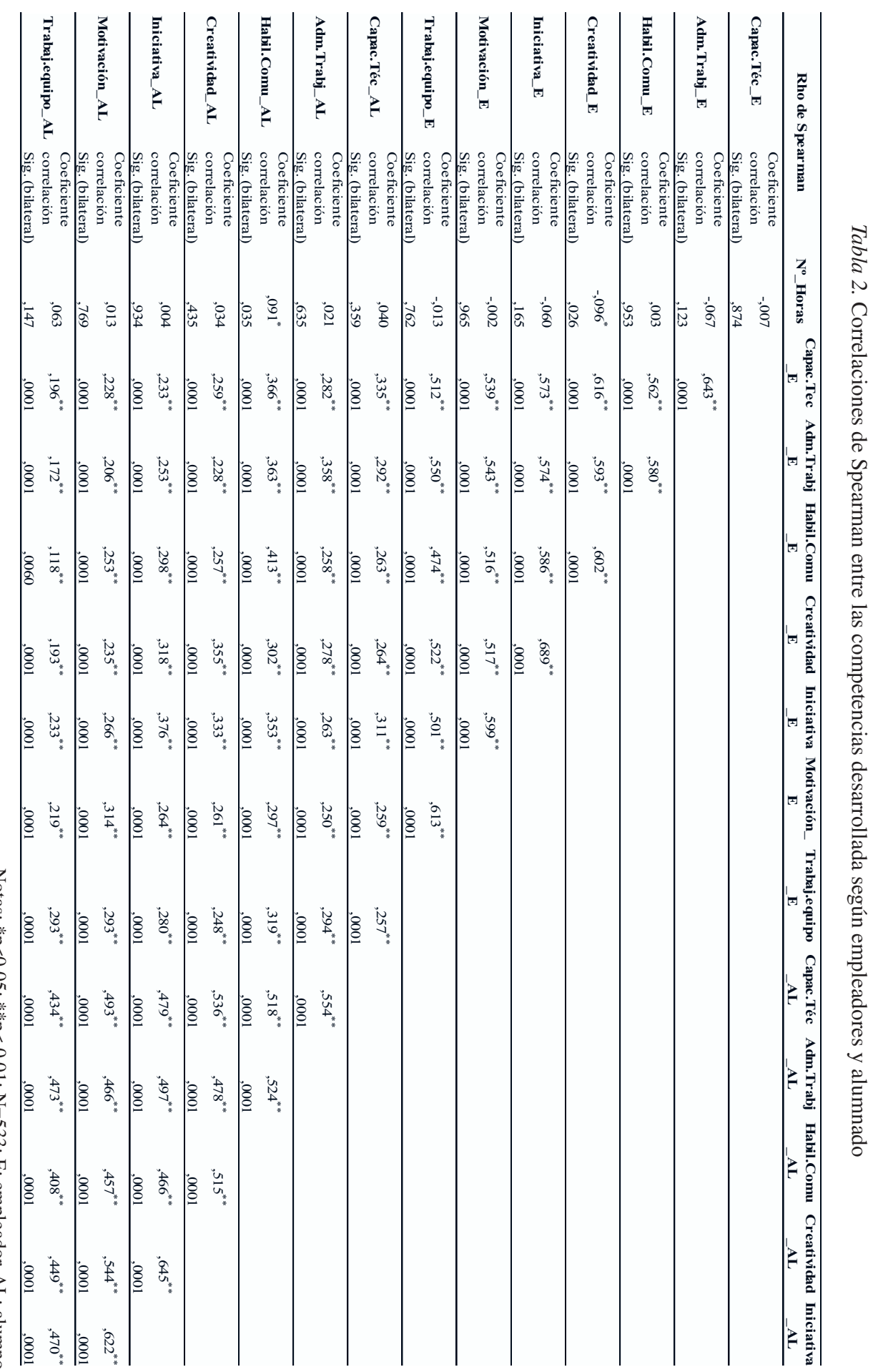


El grado cursado es la segunda variable considerada en el análisis, siendo los resultados muy similares en ambos grados, aunque como se observa en la Tabla 4 aparecen diferencias en opinión del alumnado en iniciativa personal y en el trabajo en equipo, siendo mayor en el grado de Turismo.

La tercera variable estudiada es el curso académico, no existiendo diferencias, por lo que, las opiniones del alumnado y de los empleadores son similares en los cuatro años analizados.

Tabla 4. Resultados de la prueba U Mann-Whitney sobre las competencias desarrolladas según el alumnado en función del grado

\begin{tabular}{|l|l|c|c|r|c|}
\hline \multicolumn{2}{|c|}{} & N & Rango promedio & Suma rangos & Sig. \\
\hline \multirow{2}{*}{ Iniciativa-AL } & ADE & 319 & 254,67 & 81239,00 & \multirow{2}{*}{011} \\
\cline { 2 - 6 } & Turismo & 214 & 285,38 & 61072,00 & \\
\hline \multirow{2}{*}{ Trabajo en equipo-AL } & ADE & 319 & 256,71 & 81890,00 & \multirow{2}{*}{, 014} \\
\cline { 2 - 6 } & Turismo & 214 & 282,34 & 60421,00 & \\
\hline
\end{tabular}

Notas: N=533; AL: alumno

Finalmente, la cuarta variable considerada es la duración de las prácticas, medida a través del número de horas realizadas. Se agruparon el número de horas en tres categorías o intervalos y no se encuentran diferencias salvo en la opinión del alumnado con respecto a las habilidades de comunicación oral y escrita donde parece ser que el dominio de esta capacidad será mayor cuanto mayor es el intervalo de horas de prácticas (Tabla 5).

Tabla 5. Resultados de la prueba H Kruskal-Wallis sobre las competencias desarrolladas según el alumnado en función de la duración de las prácticas

\begin{tabular}{|l|l|c|c|c|}
\hline \multicolumn{2}{|c|}{} & N & Rango promedio & Sig. \\
\hline \multirow{3}{*}{$\begin{array}{l}\text { Habilidades comunicación } \\
\text { oral y escrita-AL }\end{array}$} & 150 horas & 115 & 252,44 & \\
\cline { 2 - 4 } & Entre 151 y 300 horas & 321 & 263,25 & \multirow{2}{*}{040} \\
\cline { 2 - 4 } & 301 o más horas & 97 & 296,65 & \\
\hline
\end{tabular}

Notas: $\mathrm{N}=533$; AL: alumno

La clasificación de los intervalos realizada en esta variable se basa en el número de horas que habitualmente realiza el alumnado. Generalmente, estos realizan un mínimo de 150 horas, entre 150 y 300 o más de 300, estas últimas más habituales cuando realizan planes de carrera que suelen tener una duración de entre 3 y 6 meses. 


\subsection{RESULTADOS DEL ANÁLISIS DE LAS MEMORIAS DE VERIFICACIÓN DE LOS GRADOS}

Como se puede observar en la Tabla 6 solamente se hace referencia en ambas titulaciones a la iniciativa, motivación, trabajo en equipo y habilidades de comunicación oral y escrita. Se añade creatividad en Turismo. En ADE, la motivación e iniciativa se clasifican como otras competencias y el trabajo en equipo y las habilidades de comunicación oral y escrita se categorizan como genérales y específicas. En turismo, la creatividad, motivación e iniciativa se incluyen como otras competencias y la capacidad de comunicación oral y escrita y la capacidad de trabajo en equipo se clasifican en genérales y específicas. No se diferencia entre ambas, por lo que se demuestra la ambigüedad y la dificultad para identificar las competencias en los diseños de los diferentes títulos académicos, así como en algunos casos la falta de concordancia con el listado de competencias a evaluar en los informes de prácticas, elaborados por la propia universidad para todas las titulaciones.

Tabla 6. Inclusión en la memoria de verificación de los grados de las competencias transversales a desarrollar

\begin{tabular}{|l|c|c|c|c|}
\hline \multirow{2}{*}{} & \multicolumn{2}{|c|}{ ADE } & \multicolumn{2}{c|}{ TURISMO } \\
\cline { 2 - 5 } & Si & No & Si & No \\
\hline Capacidad técnica & $\mathrm{X}$ & & $\mathrm{X}$ & \\
\hline Administración de trabajos & & $\mathrm{X}$ & & $\mathrm{X}$ \\
\hline Habilidades comunicación oral y escrita & $\mathrm{X}$ & & $\mathrm{X}$ & \\
\hline Creatividad & & $\mathrm{X}$ & $\mathrm{X}$ & \\
\hline Iniciativa & $\mathrm{X}$ & & $\mathrm{X}$ & \\
\hline Motivación & $\mathrm{X}$ & & $\mathrm{X}$ & \\
\hline Trabajo en equipo & $\mathrm{X}$ & & $\mathrm{X}$ & \\
\hline
\end{tabular}

Con respecto a la capacidad técnica en la memoria de verificación se incluyen las siguientes:

En ADE:

- Capacidad de análisis y síntesis.

- Capacidad de desempeño efectivo dentro de un equipo de trabajo.

- Capacidad de aprendizaje y trabajo autónomo.

- Capacidad de aplicar los conocimientos teóricos y prácticos adquiridos en un contexto académico especializado.

En Turismo:

- Capacidad para interpretar críticamente datos y texto.

- Capacidad de aprendizaje y trabajo autónomo.

- Capacidad de aplicar conocimientos prácticos y teóricos adquiridos en un contexto 
académico especializado.

- Capacidad para convertir un problema empírico en un objeto de investigación y elaborar conclusiones.

- Capacidad de trabajo en equipo, así como a nivel individual.

Si se analizan estas capacidades se observa que podrían entenderse como transversales ya que, salvo la capacidad de aplicar conocimientos prácticos y teóricos adquiridos en un contexto académico especializado, las demás no tienen una relación directa con habilidades propias de un entorno laboral concreto ni para el grado en Turismo ni en ADE.

\section{DISCUSIÓN Y CONCLUSIONES}

En relación al objetivo planteado en este estudio, análisis de las percepciones de los empleadores y de los estudiantes universitarios sobre la capacidad técnica y el nivel de desarrollo de las competencias transversales en su primera toma de contacto con el mundo laboral, establecida a través de las prácticas externas, se ha podido observar con el análisis realizado que, tanto el alumnado como los empleadores no cambian sustancialmente sus puntos de vista sobre el dominio de las diferentes competencias. Estos resultados son similares al estudio de Fortin y Legault (2010) quienes también llegan a la conclusión de que los empleadores generalmente consideran a los aprendices como altamente competentes o excelentes.

De hecho, en el presente estudio la opinión de los estudiantes y empleadores sobre el dominio de sus competencias transversales es realmente alta, destacando la capacidad de trabajo en equipo. Resultados similares obtienen en su estudio Martínez-Pellicer, GarcíaPalma y Llamas (2014) donde la valoración dada por los empleadores es mayor en todas las competencias estudiadas. Además, observan que las competencias más desarrolladas durante las prácticas externas coinciden entre estudiantes y empleadores, existiendo diferencias en el trabajo en equipo y, siendo menores las diferencias en la preocupación por la calidad. Clemente-Ricolfe y Escribá-Pérez denotan que el alumnado piensa que las competencias genéricas más adquiridas son la capacidad de análisis, la resolución de problemas y el trabajo en equipo, y consideran que debería mejorarse el conocimiento de idiomas extranjeros y la capacidad de negociación, por lo que sería importante añadir estas habilidades al elenco de competencias de las titulaciones estudiadas (Clemente-Ricolfe \& Escribá-Pérez, 2013). Por tanto, la evidencia sugiere que el trabajo en equipo es esencial para una práctica segura y confiable. Riebe, Roepen, Santarelli y Marchioro señalan que el trabajo en equipo se puede mejorar proporcionando un marco conceptual claro para desarrollar las habilidades de interacción grupal (Riebe, Roepen, Santarelli \& Marchioro, 2010).

No existen diferencias entre la opinión de los empleadores y de los estudiantes con respecto al dominio de sus habilidades de comunicación. Sin embargo, la percepción competencial de los empleadores si es mayor que la del alumnado en relación a su capacidad técnica, administración de trabajos, creatividad, iniciativa, motivación y trabajo en equipo. Este resultado es avalado en el análisis correlacional dado que parece no existir unanimidad entre la opinión de los estudiantes y de los empleadores con respecto al dominio de competencias ya que, aunque las correlaciones son positivas y significativas son realmente bajas. Ello puede indicar la existencia de correlaciones no lineales, por 
lo que sería importante realizar un estudio donde se incorporasen variables de carácter personal o sociodemográfico que pudiesen explicar estos resultados.

En un trabajo similar en la Universidad de La Coruña, Freire et al. intentan analizar la concordancia con las competencias requeridas por los empresarios y las trabajadas en las diferentes titulaciones de la universidad, llegando estos investigadores a la conclusión, en términos generales, que la adecuación entre estos indicadores es media (Freire et al., 2013). También en el estudio de Acedo et al., se señala la existencia de discrepancias entre las habilidades adquiridas por los estudiantes durante su formación en la universidad y las habilidades prácticas necesarias para la realización de su trabajo en las diferentes empresas (Acedo et al., 2017). Estos autores sugieren que la capacitación ofrecida por las universidades no siempre es suficiente o está orientada a las demandas que exigen, en la actualidad, tanto la sociedad como el mercado laboral. En esta línea, Cabrerá et al. afirman la existencia de cierta escisión entre el mundo académico y el ámbito empresarial, ya que este último valora en mayor medida las competencias generales, y por el contrario la formación universitaria incide más en las competencias específicas (Cabrerá et al., 2016). Estos autores observan que los empleadores valoran, principalmente, las competencias genéricas, concretamente las de carácter interpersonal (entre las más valoradas, el trabajo en equipo) y sistémicas (el liderazgo) y, en menor medida, las instrumentales.

En España, la normativa actual no ha sido ajena a estas propuestas europeas ya que, al formar parte de los países que se sumaron al plan europeo de convergencia de la educación superior, ha adoptado su normativa (Sánchez-Elvira et al., 2010). Tanto la publicación del Documento Marco por el Ministerio de Educación, Cultura y Deporte (MECD, 2003) como la formalización de las nuevas enseñanzas del Real Decreto 1393/2007 (BOE, 2007), que establece la ordenación de las enseñanzas universitarias oficiales, mencionan en lo referente a las competencias y a las nuevas metodologías, que los objetivos formativos deben incluir competencias más específicas que ofrezcan orientación profesional y conduzcan a la integración posterior en el mercado de laboral (MECD, 2003).

De hecho, en el presente estudio destaca el bajo nivel de creatividad percibido tanto por los empleadores como por los estudiantes a pesar de ser patente, en los últimos años, la importancia de esta capacidad para el rendimiento académico (Naderi, Abdullah, Aizan, Sharir \& Kumar, 2010). Cabe destacar que, si bien no se observan diferencias significativas en creatividad a lo largo de los cuatro cursos analizados, esta competencia ha experimentado una mejora sustancial, y más evidente desde la percepción del alumnado a medida que avanzan los cursos académicos. Esto puede ser debido a ciertas iniciativas llevadas a cabo desde la dirección del centro analizado (Facultad de Ciencias Empresariales y Turismo de Ourense) iniciadas en el curso 2013/14, donde ya se había detectado una carencia importante en esta competencia (Mareque, De Prada \& Rivo, 2015). De todas formas, al no ser significativa parece ser que, o bien dichas iniciativas no han sido suficientes o todavía no han empezado a dar sus frutos.

Por otra parte, también se ha observado que según los empleadores el índice de creatividad es menor cuantas más horas de prácticas realiza el alumnado. Esto podría ser debido a que los estudiantes decidan resolver las cuestiones por algún tipo de protocolo establecido en la empresa y dejan por tanto de ofrecer alternativas a las dinámicas que se van planteando en las tareas diarias de su puesto laboral. Desde la perspectiva de los estudiantes, a mayor número de horas de prácticas mayores capacidades en habilidades comunicativas, es decir, el alumnado percibe que de las distintas competencias desarrolladas son las 
habilidades de comunicación oral y escrita donde mejoran cuanto mayor es la duración de la práctica. Martínez-Pellicer et al. destacan que un factor fundamental para el proceso de aprendizaje competencial es la duración de las prácticas, pues parece lógico pensar que el nivel de las competencias debe mejorar a mayor número de horas realizadas (MartínezPellicer et al., 2014). Además, obtienen resultados similares a este estudio, observando que el alumnado percibe que las habilidades en comunicación oral y escrita son superiores cuando realizan prácticas de duración entre 301 y 500 horas que cuando realizan prácticas inferiores a 300 horas.

En los últimos años, un importante número de estudios han destacado la importancia de las destrezas/competencias no técnicas (denominadas como soft skills) como esenciales para el éxito profesional, lo que se ha traducido en el énfasis de su importancia por parte de las autoridades europeas y su consecuente integración en los programas de grado universitarios en forma de competencias transversales. Entre estas destrezas se erigen como esenciales las de índole comunicativo (cf. Wesley, Jackson \& Lee, 2017). Estos autores tras un estudio realizado con el fin de medir la importancia otorgada a las soft skills dentro del contexto universitario en estudiantes en especialidades de gestión, concluyen que tanto para el alumnado como para profesores y empleadores las destrezas de comunicación son las más valoradas para su ejercicio profesional. Además, es importante señalar la sólida relación que existe entre la creatividad y las habilidades de escritura, prácticas esenciales para la consecución del dominio de competencias de comunicación escrita, que deben ser especialmente fomentadas, ya que aúnan dos elementos esenciales de las demandas empresariales, competencias en comunicación y creatividad. En esta línea, Ortiz, RegionSebest y MacDermott manifiestan que las habilidades de comunicación más exigidas por las empresas son el uso adecuado de la gramática, la comunicación entre el equipo, la capacidad de entablar conversación, la participación en la reunión y, la capacidad de hablar bien por teléfono (Ortiz, Region-Sebest \& MacDermott, 2016). Respecto a las habilidades de comunicación escritas, Flaherty y Choi concluyen en su estudio que la percepción del alumnado es que la escritura es menos importante que las habilidades orales e interpersonales para los puestos de primera línea en una empresa (Flaherty \& Choi, 2014). Pocos empleadores seleccionan y miden las habilidades de escritura durante la etapa de reclutamiento y selección y, solamente crece la necesidad de habilidades de escritura a medida que avanza la carrera profesional.

Desde el punto de vista del género, los empleadores opinan que las mujeres tienen mayor capacidad de administrar trabajos y de habilidades de comunicación, en cambio el alumnado opina que es mayor su iniciativa personal. De todas formas, cabe destacar que las puntuaciones otorgadas en cada una de las competencias analizadas son mayores en las mujeres, resultado similar al que obtenían Freire et al. (2013), donde de las diecinueve competencias valoradas solo en tres de ellas, capacidad de análisis y síntesis, habilidad para trabajar de forma independiente y resolución de problemas, los varones obtenían puntaciones superiores.

En cuanto al grado cursado, el alumnado opina que la titulación de Turismo tiene un mayor dominio en iniciativa personal y en trabajo en equipo. García-Manjón \& PérezLópez (2008) señalan que se debe prestar mayor atención al desarrollo de competencias de planificación y gestión de la empresa turística en coordinación con el desarrollo de competencias lingüísticas, las tecnologías de la información y comunicación y las específicas en cada ámbito de especialidad, dado que las competencias de planificación 
y gestión son las que muestran mayor número de interrelaciones con otros bloques competenciales. Martínez-Pellicer et al. (2014) observan que el alumnado de los grados del área jurídico-social alcanzan medias más bajas en las competencias analizadas que el resto de las áreas de conocimiento, destacando el alumnado de Ciencias de la Salud, los cuales obtienen las más elevadas a excepción de las competencias en autonomía y en resolución de problemas donde es superior en el alumnado de Humanidades. En función de estos datos podemos concluir que existen pequeñas brechas entre la opinión de los empleadores y el alumnado dependiendo de las titulaciones.

Resultaría imprescindible que durante el diseño de los planes de estudio de las titulaciones se realizase un estudio con el fin de determinar cuáles son las competencias que el alumnado debe dominar para su desempeño profesional y adecuar esa selección convenientemente a uno u otro tipo de competencia según la normativa legal vigente. Wesley et al. señalan la necesidad de combinar cualidades personales como la comunicación o liderazgo con habilidades sociales para un desempeño profesional exitoso en un mundo empresarial muy cambiante y ponen de relieve la importancia de incorporar estas capacidades en los planes de estudios (Wesley et al., 2017). Palmer, Montaño y Palou llegan a la conclusión de que en diferentes grados universitarios se detecta una escasa presencia de competencias relacionadas con el desarrollo personal y social (Palmer, Montaño \& Palou, 2009). Además, es necesario analizar durante la etapa de formación universitaria el dominio de las diferentes competencias dado que los empleadores no tienen mucha confianza en el nivel de la capacidad de los graduados (Azevedo et al., 2012).

\section{IMPLICACIONES}

En función de estas conclusiones se considera que sería necesario establecer una serie de medidas que permitan corregir algunas de las dificultades detectadas, como pueden ser:

- Realizar un diseño de los planes de estudio de las titulaciones en función de estudios previos sobre cuáles son las competencias que el alumnado debe dominar para su desempeño profesional en una realidad laboral cambiante, teniendo en cuenta la opinión de los empleadores para las titulaciones de gestión.

- Realizar un análisis reflexivo sobre los diseños de las memorias de verificación de los títulos universitarios para adaptarlos a la realidad laboral de los egresados y establecer en los programas docentes contenidos y actividades que mejoren las competencias transversales, dada su importancia en el desempeño laboral.

- Fortalecer el desarrollo de competencias como la creatividad, el trabajo en equipo, las habilidades de comunicación y la iniciativa o el espíritu emprendedor dentro de las titulaciones de educación superior.

- Otorgar una mayor importancia a competencias relacionadas con las habilidades comunicativas y su relación con el ámbito internacional, relacionados con los objetivos fundamentales del EEES, tales como la movilidad internacional, la interculturalidad, basada en el desarrollo de capacidades para escribir, hablar y redactar en idiomas extranjeros o el conocimiento de la cultura y costumbres de otros países.

- Diseñar programas de formación pedagógica del profesorado universitario que le permita utilizar dinámicas y estrategias didácticas activas y creativas basadas, por 
ejemplo, en la simulación para tener un entrenamiento cuando llegue la práctica directa en la empresa y facilitar al alumnado el dominio de diferentes competencias transversales.

- Mejorar las técnicas e instrumentos de evaluación de las competencias transversales que permitan la eliminación de la subjetividad y faciliten el rigor y la retroalimentación del proceso de enseñanza-aprendizaje, y adaptarlas a las distintas áreas de conocimiento, incluyendo competencias específicas de la titulación en cuestión.

\section{REFERENCIAS BIBLIOGRÁFICAS}

Acebrón, A.M. (2008). Las competencias transversales en la Universidad Pompeu Fabra. La visión de los docentes y estudiantes de segundo ciclo. Revista de Docencia Universitaria, 1, 1-16. Recuperado el 20 de febrero de 2018 desde http://www.redu.m.es/Red_U/m1.

Acedo, M.A., Azcona, E., Riaño, C. \& Ruiz, F.J. (2017). Satisfaction with external internships: Do students acquire the professional skills necessary to improve their employability? International Journal of Human Capital and Information Technology Professionals, 8(1), 42-50. DOI: 10.4018 / IJHCITP.2017010103.

Agudo, J.E., Hernández-Linares, R., Rico, M. \& Sánchez, H. (2013). Competencias transversales: percepción de su desarrollo en el grado en ingeniería en diseño industrial y desarrollo de productos. Formación universitaria, 6(5), 39-50. Recuperado el 15 marzo de 2018 desde https://scielo.conicyt.cl/scielo.php?script=sci_arttext\&pid=S0718-50062013000500006. DOI: 10.4067/S0718-50062013000500006.

Alonso, L.E., Fernández. C.J. \& Nyssen, J.M. (2009). El debate sobre las competencias. Una investigación cualitativa en torno a la educación superior y el mercado de trabajo en España. Madrid: Agencia Nacional de Evaluación de la Calidad y Acreditación-ANECA.

Álvarez-Rojo, V. \& Lázaro, A. (Coords.) (2002). Calidad de las universidades y orientación universitaria. Málaga: Aljibe.

Argos, J. \& Ezquerra, P. (2014). Universities and skills for employability. Procedia-Social and Behavioral Sciences, 139, 290-296. Recuperado el 30 de marzo de 2018 desde https://www. sciencedirect.com/science/article/pii/S1877042814046564. DOI: 10.1016/j.sbspro.2014.08.002.

Arquero, J.L., Byrne, M., Flood, B. \& González, J.M. (2009). Motives, expectations, preparedness and academic performance: A study of students of accounting at a Spanish university, Revista de Contabilidad, 12(2), 279-299. Recuparado el 20 de enero de 2018 desde http://www.redalyc. org/pdf/3597/359733634004.pdf. DOI: 10.1016/S1138-4891(09)70009-3.

Azevedo, A., Apfelthaler, G. \& Hurst, D. (2012). Competency development in business graduates: An industry-driven approach for examining the alignment of undergraduate business education with industry requirements. The International Journal of Management Education, 10(1), 1228. Recuperado el 4 de abril de 2018 desde https://www.sciencedirect.com/science/article/pii/ S1472811712000031. DOI: 10.1016/j.ijme.2012.02.002.

Boletín Oficial del Estado (2007). Real Decreto 1393/2007, de 29 de octubre, por el que se establece la ordenación de las enseñanzas universitarias oficiales. Recuperado de https://www.boe.es/ buscar/act.php?id=BOE-A-2007-18770.

Cabrera, N., López, M. \& Portillo, M. (2016). Las competencias de los graduados y su evaluación desde la perspectiva de los empleadores. Revista de Estudios Pedagógicos, 42(3), 69-87. Recuperado el 20 de mayo de 2018 desde .http://www.redalyc.org/pdf/1735/173550019004.pdf. DOI: $10.4067 / \mathrm{S} 0718-07052016000400004$.

Clemente-Ricolfe, J.S. \& Escribá-Pérez, C. (2013). Análisis de la percepción de las competencias 
genéricas adquiridas en la universidad. Revista de Educación, 362, 535-561. Recuperado el 22 de abril de 2018 desde https://dialnet.unirioja.es/servlet/articulo?codigo=4480323. DOI:10.4438/1988-592X-RE-2013-362-241.

Flaherty, J. \& Choi, H.S.C. (2014). Is writing important for graduate success? A preliminary investigation into the communication skills required of hospitality and tourism graduates. Teaching and Learning Innovations, 16, 1-9. Recuperado el 12 de febrero de 2018 desde http:// www.criticalimprov.com/index.php/tli/article/view/2793.

Fortin, A. \& Legault, M. (2010). Development of generic competencies: Impact of a mixed teaching approach on students' perceptions. Accounting Education: an international journal, 19(1-2), 93-122. doi: 10.1080/09639280902888195.

Freire, M.J., Teijeiro, M. \& Pais, C. (2011). Políticas educativas y empleabilidad: ¿cuáles son las competencias más influyentes? Archivos Analíticos de Políticas Educativas, 19(28), 1-24. Recuperado desde http://www.redalyc.org/pdf/2750/275019735028.pdf

(2013). La adecuación entre las competencias adquiridas por los graduados y las requeridas por los empresarios. Revista de Educación, 362, 13-41. Recuperado el 15 de abril de 2018 desde https://www.mecd.gob.es/revista-de-educacion/numeros-revista-educacion/numerosanteriores/2013/re362/re362_01.html. DOI: 10.4438/1988-592X-RE-2011-362-151.

Gallifa, J. \& Garriga, J. (2010). Generic competences in higher education: Studying their development in undergraduate social science studies by means of a specific methodology. Perspectives in Education, 28(3), 70-86.

García-Manjón, J.V. \& Pérez-López, M.C. (2008). El grado en turismo: un análisis de las competencias profesionales. Cuadernos de Turismo, 21, 67-83. Recuperado el 20 de marzo de 2018 desde http://revistas.um.es/turismo/article/view/24991/24281.

Gil, J. (2007). La evaluación de competencias laborales. Educación XX1, 10, 83-106. Recuperado el 25 de marzo de 2018 desde http://www.redalyc.org/html/706/70601006/.

Gliem, J. \& Gliem. R. (2003). Calculating. Interpreting. and reporting Cronbach's alpha reliability coefficient for likert-type scales. Conference in Adult. Continuing and Community Education, Midwest Research to Practice, Columbus, The Ohio State University. Recuperado el 2 de febrero de 2018 desde https://scholarworks.iupui.edu/bitstream/handle/1805/344/gliem+\&+gliem. pdf?sequence $=1$.

Huete, R. (2007). Tourism studies in Spain. The Role of Sociology in Degree Programmes. Journal of Teaching in Travel and Tourism, 7(2), 73-92. DOI: 10.1300/J172v07n02_05

Jackson, D. (2012). Business undergraduates' perceptions of their capabilities in employability skills: Implications for industry and higher education. Industry and Higher Education, 26(5), 345356. Recuperado el 20 de enero de 2018 desde http://journals.sagepub.com/doi/abs/10.5367/ ihe.2012.0117. DOI: $10.5367 /$ ihe.2012.0117

Jackson, D., \& Chapman, E. (2012a). Empirically derived competency profiles for Australian business graduates and their implications for industry and business schools. The International Journal of Management Education, 10(2), 112-128. Recuperado el 7 de febrero de 2018 desde https://www. sciencedirect.com/science/article/pii/S1472811712000237. DOI: 10.1016/j.ijme.2012.04.002.

. (2012b). Non-technical competencies in undergraduate business degree programs: Australian and UK perspectives. Studies in Higher Education, 37(5), 541-567. doi:10.1080/03075079.20 10.527935 .

Kellermann, P. (2007). Acquired Competences and Job Requirements. In U. Teichler (Ed.), Careers of university graduates: views and experiences in comparative perspectives (pp. 115-130). The Netherlands: Springer.

Kirkwood-Tucker, T.F. (2004). Toward a European model of higher Education: Processes, problems, and promises. European Education, 36(3), 51-69. DOI: 10.1080/10564934.2004.11042366.

Lans, T., Blok, V. \& Wesselink, R. (2014). Learning apart and together: towards an integrated competence framework for sustainable entrepreneurship in higher education. Journal of 
Estudios Pedagógicos XLIV, N 3: 137-155, 2018

ESTUDIO SOBRE LA CAPACIDAD TÉCNICA Y LAS COMPETENCIAS TRANSVERSALES DESARROLLADAS EN

LAS PRÁCTICAS EXTERNAS UNIVERSITARIAS

Cleaner Production, 62, 37-47. Recuperado el 5 de marzo de 2018 desde https://ac.els-cdn.com/ S0959652613001741/1-s2.0-S0959652613001741-main.pdf?_tid=6496ed41-4a87-47b5-a4e2ffdac971052e\&acdnat=1529390147_76cd82ef6fe71a86421c29c207d3f8e5. DOI: $10.1016 / j$. jclepro.2013.03.036.

Lantarón, B. (2014). La empleabilidad en la universidad española. Journal for Educators, Teachers and Trainers, 5(2), 272 -286. Recuperado el 23 de abril de 2018 desde http://jett.labosfor.com/ index.php/jett/article/view/98/98

López-Bonilla, J.M. \& López-Bonilla, L.M. (2014). Holistic competence approach in tourism higher education: An exploratory study in Spain. Current Issues in Tourism, 17(4), 312-326. DOI: 10.1080/13683500.2012.720248.

Mareque, M. \& De Prada, E. (2018). Evaluación de las competencias profesionales a través de las prácticas externas: incidencia de la creatividad. Revista de Investigación Educativa, 36(1), 203-219. Recuparado el 5 de mayo de 2018 desde http://revistas.um.es/rie/article/ viewFile/275651/221601. DOI: 10.6018/rie.36.1.275651.

Mareque, M., De Prada, E. \& Rivo, E. (2015). Análisis y evaluación por parte de los estudiantes y de las empresas sobre las competencias adquiridas en las prácticas externas. XIII Symposium internacional sobre practicum y las prácticas externas. Documentar y evaluar la experiencia de los estudiantes en las prácticas. Poio (Pontevedra), 29-30 junio y 1 de julio.

Martínez-Pellicer, A., García-Palma, M.B. \& Llamas A. (2014). El desarrollo de competencias profesionales transversales en las prácticas extracurriculares. Evaluación comparativa de alumnos y tutores. Procedia - Social and Behavioral Sciences, 139, 251-259. Recuperado el 20 de abril de 2018 desde http://www.revistaeducacion.mec.es/doi/362_151.pdf. DOI: 10.1016/j. sbspro.2014.08.070.

Mateo, J. \& Vlachopoulos, D. (2013). Reflexiones en torno al aprendizaje ya la evaluación en la universidad en el contexto de un nuevo paradigma para la educación superior. Educación XX1, 16(2), 183-208. Recuperado el 23 de febrero de 2018 desde http://www.redalyc.org/ pdf/706/70626451010.pdf. doi:10.5944/educxx1.16.2.2639.

Ministerio de Educación, Cultura y Deporte (2003). La integración del sistema universitario español en el Espacio Europeo de Educación Superior. Documento Marco. Recuperado de http://www. eees.es/pdf/Documento-Marco_10_Febrero.pdf.

Montoro, E.Mª Mora, M. \& Ortiz de Urbina, M. (2012). Análisis de las competencias adquiridas en los estudios de Dirección de Empresas y su grado de aplicación en las prácticas en empresas. Revista Complutense de Educación, 23(1), 241-263. Recuperado el 22 de marzo de 2018 desde https://revistas.ucm.es/index.php/RCED/article/viewFile/39112/37725. DOI: $10.5209 /$ rev_ RCED.

Mulder, M., Gulikers, J., Biemans, H. \& Wesselink, R. (2009). The new competence concept in higher education: error or enrichment? Journal of European Industrial Training, 33(8-9), 755-770. Recuperado el 7 de abril de 2018 desde https://www.emeraldinsight.com/doi/ full/10.1108/03090590910993616. DOI: 10.1108/03090590910993616.

Munar, A.M. (2007). Is the Bologne process globalizing tourism education? Journal of Hospitality, Leisure, Sport and Tourism Education, 6(2), 68-82. DOI: 10.3794 / johlste.62.164

Munar, A.M. \& Montaño, J.J. (2009). Generic competences and tourism graduates. Journal of Hospitality, Leisure, Sport and Tourism Education, 8(1), 70-84. DOI: 10.3794/johlste.81.206.

Naderi, H., Abdullah, R., Aizan, H.T., Sharir, J. \& Kumar, V. (2010). Relationship between creativity and academic achievement: A study of gender differences. Journal of American Science, 6(1), 181-190. Recuperado el 6 de febrero de 2018 desde http://www.jofamericanscience.org/journals/ am-sci/am0601/24_1104_Relationship_am0601.pdf.

Organización para la Cooperación y el Desarrollo Económicos (2002). Definition and selection of competentes. DESECO. Theoretical and conceptual foundation, strategy paper. Recuperado de http://oecd.org. 
Ortiz, L.A., Region-Sebest, M. \& MacDermott, C. (2016). Employer perceptions of oral communication competencies most valued in new hires as a factor in company success. Business and Professional Communication Quarterly, 79(3), 317-330. Recuperado el 20 de marzo de 2018 desde http://journals.sagepub.com/doi/abs/10.1177/2329490615624108. DOI: $10.1177 / 2329490615624108$.

Palmer, A., Montaño, J. J. \& Palou, M. (2009). Las competencias genéricas en la educación superior. Estudio comparativo entre la opinión de empleadores y académicos. Psicothema, 21(3), 433438. Recuperado el 15 de febrero de 2018 desde http://www.psicothema.com/psicothema. asp?id=3650.

Parris, M. \& Saville, K. (2011). Piecing together the puzzle of graduate employment: Factors that shape the graduate work expectations of human resource management students. Industry and Higher Education, 25(1), 15-24. Recuperado el 2 de abril de 2018 desde http://journals.sagepub. com/doi/abs/10.5367/ihe.2011.0024. DOI: 10.5367/ihe.2011.0024

Riebe, L., Roepen, D., Santarelli, B. \& Marchioro, G. (2010). Teamwork: effectively teaching an employability skill. Education + Training, 52(6/7), 528-539. Recuperado el 22 de enero de 2018 desde https://www.emeraldinsight.com/doi/full/10.1108/00400911011068478. DOI: $10.1108 / 00400911011068478$.

Rue, J. (2007). Enseñar en la universidad. El EEES como reto para la educación superior. Madrid: Narcea.

Sánchez-Elvira, A., López-González, M.A. \& Fernández-Sánchez, V. (2010). Análisis de las competencias genéricas en los nuevos títulos de grado del EEES en las universidades españolas. Revista de Docencia Universitaria, 8(1), 35-73. Recuperado el 22 de marzo de 2018 desde https://dialnet.unirioja.es/servlet/articulo?codigo=4014853. DOI: 10.4995/redu.2010.6217.

Sijtsma, K. (2009). On the use, the misuse and the very limited of the Cronbach's alpha. Psychometrika, 74(1), 107-120. Recuperado el 20 de enero de 2018 desde https://www.ncbi.nlm.nih.gov/pmc/ articles/PMC2792363/. DOI: 10.1007 / s11336-008-9101-0.

Tejada, J. (2012). La alternancia de contextos para la adquisición de competencias profesionales en escenarios complementarios de educación superior: marco y estrategia. Educación XXI, 15(2), 17-40. Recuperado el 8 de marzo de 2018 desde http://www.redalyc.org/html/706/70624504010/.

Velde, C. (2009). Employers' perceptions of graduate competencies and future trends in higher ocational education in china. Journal of Vocational Education \& Training, 61(1), 35-51. doi: 10.1080/13636820902819974.

Wesley, S.C., Jackson, V.P. \& Lee, M. (2017). The perceived importance of core soft skills between retailing and tourism management students, faculty and businesses. Employee Relations, 39(1), 79-99. Recuperado el 11 de febrero de 2018 desde https://www.emeraldinsight.com/doi/ full/10.1108/ER-03-2016-0051. DOI: 10.1108/ER-03-2016-0051. 
